\title{
Correction to: The Three-Dimensional Culture of Epithelial Organoids Derived from Embryonic Chicken Intestine
}

\section{Malgorzata Pierzchalska, Malgorzata Panek, Malgorzata Czyrnek, and Maja Grabacka}

Correction to:

Methods in Molecular Biology

https://doi.org/10.1007/7651_2016_15

There are two corrections for this chapter:

In Figure 4 Section A, the upper right corner should read " $3 \mathrm{~d}$ ", whereas it was incorrectly printed as " $4 \mathrm{~d} . "$

Also, the caption of Figure 5 should read: "The cultures were photographed on the first, second and fifth day of culture ( $1 \mathrm{~d}, 2 \mathrm{~d}, 5 \mathrm{~d}$ ) with differential interference contrast optics." rather than the incorrect "The cultures were photographed on second, third, and fifth day of culture $(2 \mathrm{~d}, 3 \mathrm{~d}, 5 \mathrm{~d})$ with differential interference contrast optics".

These issues have now been corrected.

The updated online version of this protocol can be found at https://doi.org/10.1007/7651_2016_15 